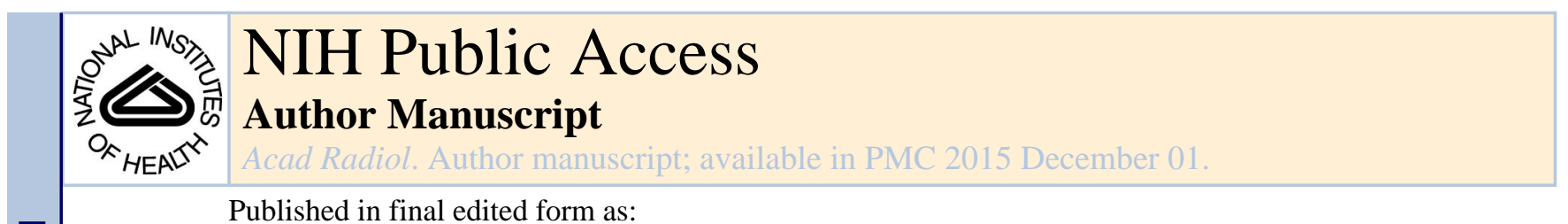

Published in final edited form as:

Acad Radiol. 2014 December ; 21(12): 1547-1552. doi:10.1016/j.acra.2014.07.009.

\title{
Comparison of a stationary digital breast tomosynthesis system to magnified 2D mammography using breast tissue specimens
}

\author{
Andrew W Tucker, PHD*, Jabari Calliste, MS, Emily M Gidcumb, BS, Jaclyn Wu, Cherie M \\ Kuzmiak, DO, Noorie Hyun, MS, Donglin Zeng, PHD, Jianping Lu, PHD, Otto Zhou, PHD, \\ and Yueh Z Lee, MD, PHD \\ University of North Carolina at Chapel Hill, Chapel Hill NC 27599
}

\section{Introduction}

Malignant lesions in the breast can be difficult to visualize using full field digital mammography (FFDM) when significant tissue overlap is present. Tissue overlap is most apparent in breast tissue that is thick and radiographically dense. Digital breast tomosynthesis (DBT) uses a small number of angular projections to reconstruct a pseudo-3D volume. Recent studies have shown an increase in the area under the curve (AUC) when using a combination of DBT and FFDM compared to FFDM alone $(1,2)$. Recall rates for benign cases significantly decrease when using a combination of DBT and FFDM (1-4). However, for cases with microcalcifications (MCs), the use of DBT along with a FFDM image has shown no significant improvement in the AUC (2).

Current DBT systems use a single X-ray source which is translated over an angular span that typically covers between 15 and 50 degrees (5-9). Larger angular spans sample more of the Fourier domain which reduces out-of-plane reconstruction artifacts. Translating a source over a larger angular span requires longer acquisition times which leads to patient motion artifacts (10). These single source systems also suffer from poor spatial resolution due to focal-spot blurring from the tube motion (11). We have developed a stationary digital breast tomosynthesis (s-DBT) technology that utilizes a novel multiple focal-spot X-ray source array with carbon nanotubes (CNTs) as the field emission electron source (12). This stationary approach can increase acquisition speed, and eliminate the focal spot motion. It has been shown that the s-DBT device offers significant improvement in image quality, including improved MTF and lesion conspicuity in breast phantom studies $(11,13)$.

The clinical standard of care for surgical breast biopsies requires that each specimen be imaged using magnified FFDM for surgical margin assessment before the surgery is ended. Due to the limitations of FFDM, including superimposition of tissues, surgical margin

(C) 2014 The Association of University Radiologists. Published by Elsevier Inc. All rights reserved.

*Corresponding author email address and phone number: awtucker@email.unc.edu, 1-704-614-2640. Current Address: Room 269 Phillips Hall, 120 East Cameron Ave, Chapel Hill, NC 27599

Publisher's Disclaimer: This is a PDF file of an unedited manuscript that has been accepted for publication. As a service to our customers we are providing this early version of the manuscript. The manuscript will undergo copyediting, typesetting, and review of the resulting proof before it is published in its final citable form. Please note that during the production process errors may be discovered which could affect the content, and all legal disclaimers that apply to the journal pertain. 
assessment for some lesions can be impossible. FFDM is also incapable of visualizing margins which are parallel to the detector. If more accurate surgical margin assessment could be achieved, the surgical biopsy recall rate could be reduced. For this study, we sought to compare the CNT-based s-DBT system to a FFDM system in a study of breast specimens. Radiologists' evaluation of image quality was assessed through a reader study. This study represents the first human tissue imaging with the novel CNT-based device. We hypothesized that in using the s-DBT system, we will generate clinically useful tomographic images of breast specimens that are of comparable quality to conventional high magnification 2D specimen radiographs.

\section{Materials and Methods}

Informed consent was obtained for thirty-nine patients with known breast lesions (BIRADS 4 or 5), as determined by conventional screening and diagnostic mammography, and scheduled for needle localization biopsy under an institutional review board-approved protocol. After excision from the patient, the specimen was placed in a standard quasiradiolucent specimen container and compressed using the container's own compression mechanism with enough pressure to prevent the tissue from sliding in the container using a perforated grid. Figure 1 (Left) shows a 2D radiograph of an empty specimen container. An average specimen thickness of $16 \mathrm{~mm}$ after compression was observed. Specimens were imaged using a Senographe Essential FFDM system (General Electric Fairfield, CT USA) using $26 \mathrm{kVp}, 1.8 \mathrm{x}$ radiographic magnification, and a dose proportional to the specimen's size. After standard of care clinical imaging, all specimens were transported to our research facility and re-imaged using an s-DBT system. The specimens were then transported to the Department of Pathology in the hospital for standard clinical pathology evaluation. All specimens were returned to the Department of Pathology within one hour after excision (cold ischemia time). Of the 39 patients, 25 were later determined to have malignant lesions excised through pathological analysis.

The s-DBT system consists of a linear array of CNT-based X-ray sources retrofitted to a Selenia Dimensions DBT system (Hologic Bedford, MA USA). Figure 1 (Right) shows an sDBT system with a specimen container on the detector housing. The array contains 31 equiangular spaced X-ray sources covering a total span of 30 degrees with a $1 \mathrm{~mm}$ thick aluminum window. Specimens were imaged using 15 projection images distributed over 28 degrees, $1.08 \mathrm{x}$ radiographic magnification, $26 \mathrm{kVp}, 100 \mathrm{mAs}$, and a detector pixel size of 70 $\mu \mathrm{m}$. The spatial resolution of this system is $30 \%$ higher ( 4 cycles $/ \mathrm{mm}$ compared to 3 cycles $/ \mathrm{mm}$ ) than a continuous-motion DBT system (11). Projection images were reconstructed into a 3D volume using a back-projection filtering method developed by Real Time Tomography, LLC (Villanova, PA USA) (14). Images were reconstructed using a 0.5 $\mathrm{mm}$ distance between slices and a pixel size equivalent to that of a 1.8x magnified image (the magnification used for the 2D radiograph).

A reader study was performed with four breast fellowship-trained radiologists over two separate sessions; all images were viewed in each session. During the initial session, half of the specimens were viewed using 2D mammography first, and half using s-DBT first. For the second session, the readers were shown the images in reverse order. A four week wash- 
out time was given between the two sessions. The readers gave a malignancy score between 1 and 5 ( 1 - benign, 3 - 50\% chance of malignancy, 5 - highly malignant) for both masses and MCs in the specimen. The numeric malignancy score was not based on BIRADS. After malignancy scores were recorded, the second modality was shown to the reader to determine the reader's preference between s-DBT and mammography. Reader preference was recorded between -3 and +3 in increments of one ( -3 - 2D preferred, 0 - equally preferred, +3 - sDBT preferred). Reader preference was recorded for three different categories for each specimen, as applicable; (1) shape/morphology of masses, (2) MC assessment, and (3) margin assessment. The imaging protocol was approved by the institutional review board before any recruitment of patients.

For statistical analysis of the recorded malignancy score for each reader the sensitivity and specificity was determined. McNemar's test was applied to compare the discordance between the two modalities. For the reader preference portion of the study, a two sided t-test was applied to test if the modalities were different. A secondary analysis was completed on the reader preference results. It was tested whether the mean reader preference was larger than zero using a linear mixed model with a random intercept effect and Wald test. It was assumed the correlation in reader preference for each specimen between any two readers was the same. Given that the readers in the study each gave two reads on preference (which were then averaged), the two reads were compared using Cohen's kappa to indicate the consistency of the preferences over time.

\section{Results}

Four radiologists evaluated 42 specimens from 39 patients. Three of the patients had multiple specimens, in these cases reader results were only recorded for the specimen which contained the majority of tissue. Of the 39 patients, 51\% (20/39) were BIRADS 4 and 49\% (19/39) were BIRADS 5. For the BIRADS 4 patients, $40 \%$ (8/20) were determined to be malignant through pathology results. For the BIRADS 5 patients, 89\% (17/19) were determined to be malignant through pathology results. Readers 1, 2, 3, and 4 had 11, 16, 19, and 1 years of experience practicing radiology respectively. The sensitivity and specificity of each modality was calculated for each reader. Table 1 shows the calculated values using 3 as the threshold for a positive response (interpreted as malignant). Readers 1 and 3 failed to record diagnosis for one patient and therefore only have 38 total responses. Two of the four readers recorded a higher sensitivity using s-DBT than 2D mammography. Two readers gave higher specificity values for 2D mammography, one gave a higher specificity value for s-DBT, and one reader (Reader 3) did not diagnose any specimens as benign.

To compare discordance between the two modalities, McNemar's test was applied. Table 2 shows the results of McNemar's test for each reader. In the cases of readers 1, 2, and 3, there is insignificant discordance between diagnostics based on the two modalities. In the case of reader 4 diagnostics based on two modalities are likely to be different.

A two-sided t-test was conducted to determine the reader preference for the shape/ morphology of masses, the results of the test are shown in Table 3. A reader preference of 
$0.07 \pm 1.34$ was recorded, where a positive value represents a preference for s-DBT. The difference in the reader preference between the two modalities for masses was insignificant.

Table 3 also shows the two-sided t-test results for reader preference for MC assessment. Overall, an average preference of $-0.70 \pm 0.95$ was recorded, where a negative value represents a preference for 2D mammography. Figure 2 shows reconstruction slices and the corresponding 2D image of a specimen with a suspicious cluster of MCs. With the high spatial resolution of s-DBT the MCs are visible in the reconstruction.

Table 3 also shows the two-sided t-test results for reader preference with respect to surgical margin assessment. The average preference for margins was 0.16 with a standard deviation of 1.22, where a positive value represents a preference for s-DBT. Figure 3 shows an s-DBT reconstruction slice and a 2D mammography image of a specimen with a suspicious lesion with spiculated margins. Clear margin delineation is present in the s-DBT reconstruction; however, tissue overlap in the 2D image reduces margin visibility. This particular lesion was later diagnosed as malignant.

The results for the secondary analysis using the linear mixed model trended toward a preference for the s-DBT system in terms of the shape and morphology of masses and surgical margin assesment. It was found that readers preferred FFDM over s-DBT for MC visibility (p-value $<0.05$ ). The results of the secondary analysis can be found in Table 4 .

For the Cohen's kappa analysis on reader preference consistency 3 subjects with more than two reads (multiple specimens) were excluded. Also, instances where the reader did not record a reading in either the first or second session for a particular specimen were also excluded in the analysis. Inter-rater agreement of the readers varies as Cohen's kappa measurement indicates from 0.20 to 0.46 . The Cohen's kappa statistics for reader 1 and 2 is 0.20 and 0.21 ; whereas, the corresponding statistics for reader 3 and 4 are 0.46 and 0.35 , which is fairer than the readers of 1 and 2.

\section{Discussion}

Overall malignancy diagnosis of the two modalities was comparable. The prevalence of malignancy in our specimens was 25 out of 39 , due to the fact that standard of care at UNC Hospitals is to surgically remove cancer and atypia diagnosed on needle core biopsy. Thus, we would anticipate a bias toward malignant diagnosis within our specimens given the initial malignant diagnosis.

Readers trended toward a preference for s-DBT with respect to masses and surgical margins compared to magnified 2D mammography. However, magnified 2D mammography was preferred when viewing MCs. A large amount of reader fatigue was noted between the first and second session. Specifically looking at reader 1 for surgical margin assessment, it can be seen that their average preference decreased from 0.18 to -1.16 , a decrease of 1.34 . This is in contrast to all other readers whom saw an average increase of 0.51 .

Specimen radiography is essentially optimized for 2D mammography, placing s-DBT at a disadvantage for a number of key reasons. Large radiographic magnification factors are used 
that cannot be replicated on any DBT system, and the specimen container produces artifacts in s-DBT reconstructions. A typical mammography system utilizes both a large and small focal spot. The small focal spot is designated for magnification views, this reduces the effect of focal spot enlargement on image spatial resolution. Typical DBT systems (including the s-DBT system) only use a large focal spot due to power constraints on the anode. Thus for this study, a magnification factor of 1.8 was used for 2D mammography compared to 1.08 for the s-DBT images.

The specimens were held in a conventional specimen container consisting of a rectilinear grid with circular holes. The grid coordinates provide a mechanism for the communication of findings between the radiologist and the pathologist. However, in tomosynthesis the regularly spaced grids impose additional artifacts within the imaging planes of the specimen, causing image degradation. Simply removing the grid would reduce this image artifact. This was not done in this study in order to preserve the clinical workflow. If s-DBT is used in the future for specimen radiography, it would be beneficial to design a specimen container which reduced artifacts in the reconstruction images. One example could be a cone-shaped design, the specimen could be held stationary by the walls of the cone and the grid could be placed at a distance above the tissue, thereby reducing the reconstruction artifact.

Another disadvantage of s-DBT in this study was the use of biopsy needles to mark lesions (later used for localization by the pathologist). Some specimens were marked with biopsy needles after the 2D mammogram was acquired in the hospital. In some specimens a large number of needles were present in the s-DBT reconstruction and not present in the 2D mammography image. Figure 4 shows a reconstruction slice and the corresponding 2D image of a specimen which contained a substantial amount of needles. In the figure, large needle artifacts can be seen in the s-DBT reconstruction which can reduce lesion visibility. In future studies, post processing segmentation and interpolation could be used to reduce the artifacts.

In summary, we reported the first human data acquired using a stationary digital breast tomosynthesis system. Lumpectomy specimen images were acquired using a 2D mammography system and an s-DBT system. Stationary digital breast tomosynthesis was found to be comparable with 2D mammography for malignancy diagnosis but readers were significantly more confident in MC visibility when using 2D mammography ( $\mathrm{p}$-value < 0.05). Readers, with respect to masses and surgical margins, trended toward a preference for s-DBT. These results were not significant. Given the trend of preference for s-DBT over 2D mammography for both mass visibility and margin assessment, s-DBT could be a viable alternative to 2D mammography for imaging breast specimens. A future study could be designed to compare surgical margin measurements with s-DBT and magnified 2D mammography to pathological measurements. This study would quantify the added benefit of s-DBT in surgical margin assessment over magnified 2D mammography. A clinical trial is currently underway which will compare images using the s-DBT system and a 2D mammography system. 


\section{Acknowledgments}

The project is supported by the National Cancer Institute under grant number U54CA151652 and R01CA134598. We would like to thank Hologic for providing the Selenia Dimension Tomosynthesis System and for technical support. Thanks to Christy Inscoe, Jing Shan, and Marci Potuzko for support in acquiring image datasets. Thanks to Doreen Steed for help in recruiting patients.

\section{References}

1. Rafferty EA, Park JM, Philpotts LE, et al. Assessing radiologist performance using combined digital mammography and breast tomosynthesis compared with digital mammography alone: results of a multicenter, multireader trial. Radiology. 2013; 266:104-13. [PubMed: 23169790]

2. Michell M, Iqbal A, Wasan R, et al. A comparison of the accuracy of film-screen mammography, full-field digital mammography, and digital breast tomosynthesis. Clinical radiology. 2012; 67:97681. [PubMed: 22625656]

3. Bernardi D, Ciatto S, Pellegrini M, et al. Prospective study of breast tomosynthesis as a triage to assessment in screening. Breast cancer research and treatment. 2012; 133:267-71.10.1007/ s10549-012-1959-y [PubMed: 22270938]

4. Poplack SP, Tosteson TD, Kogel CA, et al. Digital breast tomosynthesis: initial experience in 98 women with abnormal digital screening mammography. AJR American journal of roentgenology. 2007; 189:616-23.10.2214/AJR.07.2231 [PubMed: 17715109]

5. Bissonnette M, Hansroul M, Masson E, et al. Digital breast tomosynthesis using an amorphous selenium flat panel detector. Proc SPIE. 2005; 5745:529-40.

6. Maidment A, Albert M, Thunberg S, et al. Evaluation of a photon-counting breast tomosynthesis imaging system. Proc SPIE. 2005:5745.

7. Ren B, Ruth C, Stein J, et al. Design and performance of the prototype full field breast tomosynthesis system with selenium based flat panel detector. Proc SPIE. 2005; 5745:550-61.

8. Ren B, Ruth C, Wu T, et al. A new generation FFDM/tomosynthesis fusion system with selenium detector. Proc SPIE. 2010; 7622:76220B.

9. Wu T, Stewart A, Stanton M, et al. Tomographic mammography using a limited number of lowdose cone-beam projection images. Med Phys. 2003; 30:365. [PubMed: 12674237]

10. Acciavatti RJ, Maidment AD. Optimization of continuous tube motion and step-andshoot motion in digital breast tomosynthesis systems with patient motion. Proc SPIE. 2012; 8313:831306-1. 12.

11. Qian X, Tucker A, Gidcumb E, et al. High resolution stationary digital breast tomosynthesis using distributed carbon nanotube X-ray source array. Med Phys. 2012; 39:2090.10.1118/1.3694667 [PubMed: 22482630]

12. Qian X, Rajaram R, Calderon-Colon X, et al. Design and characterization of a spatially distributed multibeam field emission X-ray source for stationary digital breast tomosynthesis. Med Phys. 2009; 36:4389-99. [PubMed: 19928069]

13. Tucker AW, Lu J, Zhou O. Dependency of image quality on system configuration parameters in a stationary digital breast tomosynthesis system. Med Phys. 2013; 40:031917-10. [PubMed: 23464332]

14. Kuo J, Ringer PA, Fallows SG, et al. Dynamic reconstruction and rendering of 3D tomosynthesis images. Proc SPIE. 2011; 7961:796116. 


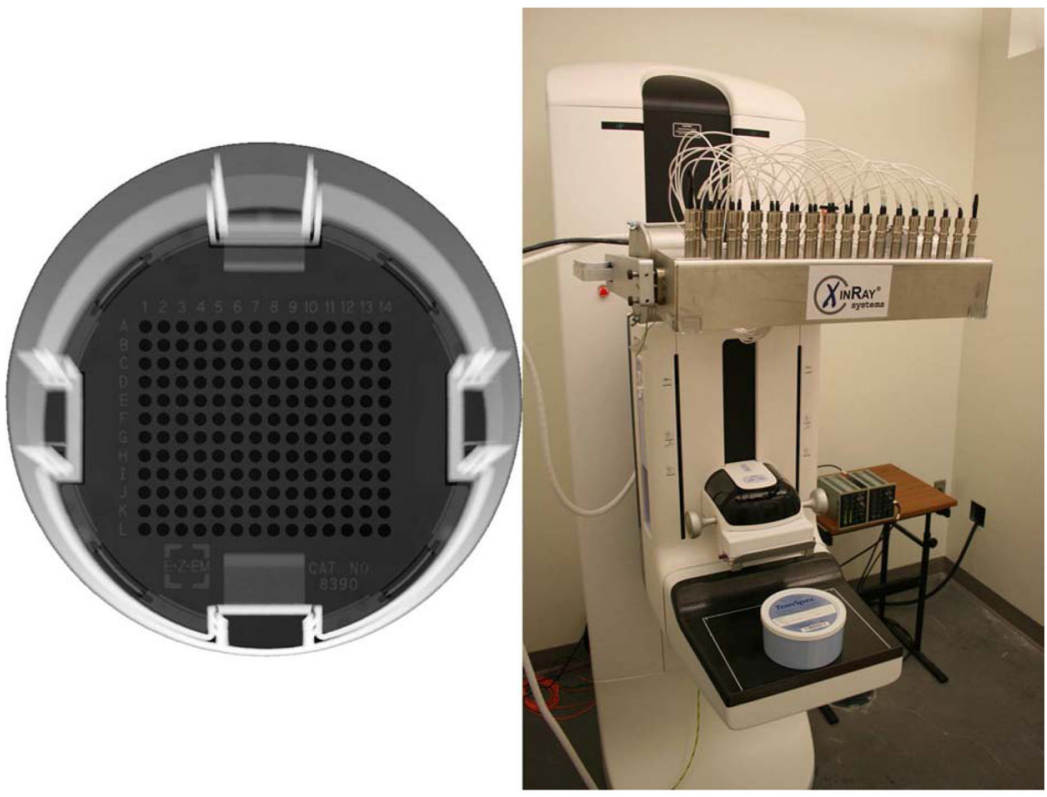

Figure 1.

Left - - Segmented 2D radiograph of container used to hold specimens. Right - Image of an s-DBT system with specimen container on the detector housing. 

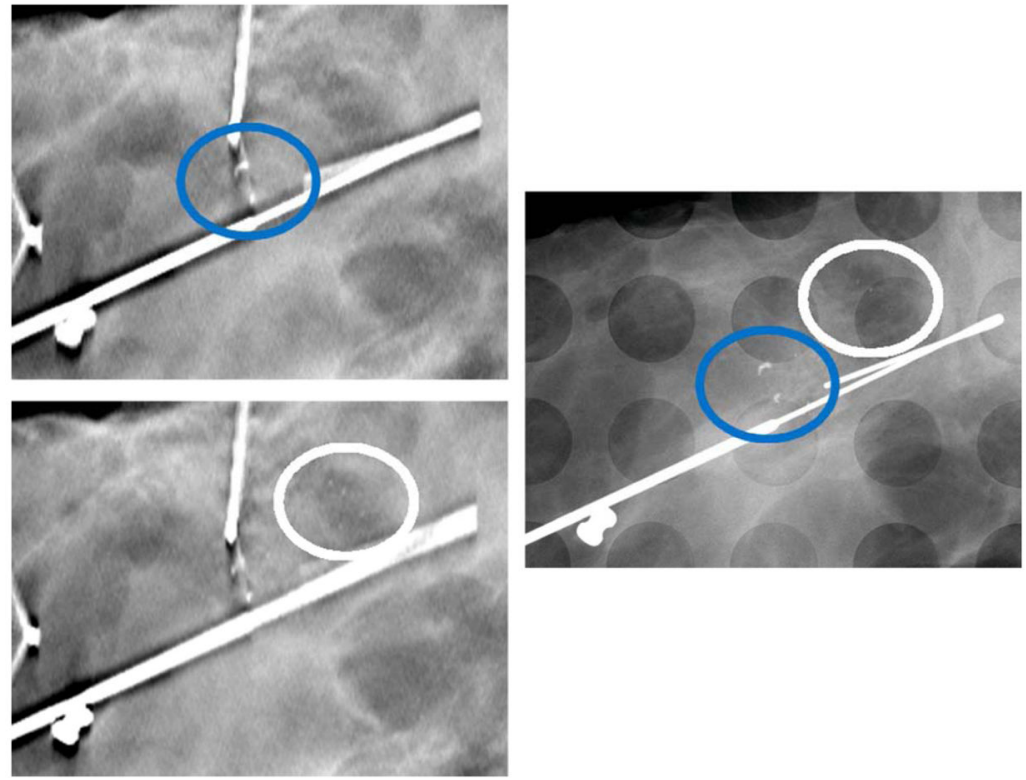

Figure 2.

Left Above - Reconstructed slice of a specimen using an s-DBT system. Left Below Reconstruction slice located $1.5 \mathrm{~mm}$ below the previous slice. Right - 2D mammography image of the same specimen. The high spatial resolution of the s-DBT system allows for imaging of small microcalcifications. The added z-axis information allows for better visualization of MC clusters. The blue oval envelopes a cluster of large MCs and the white oval envelopes a cluster of smaller MCs. 


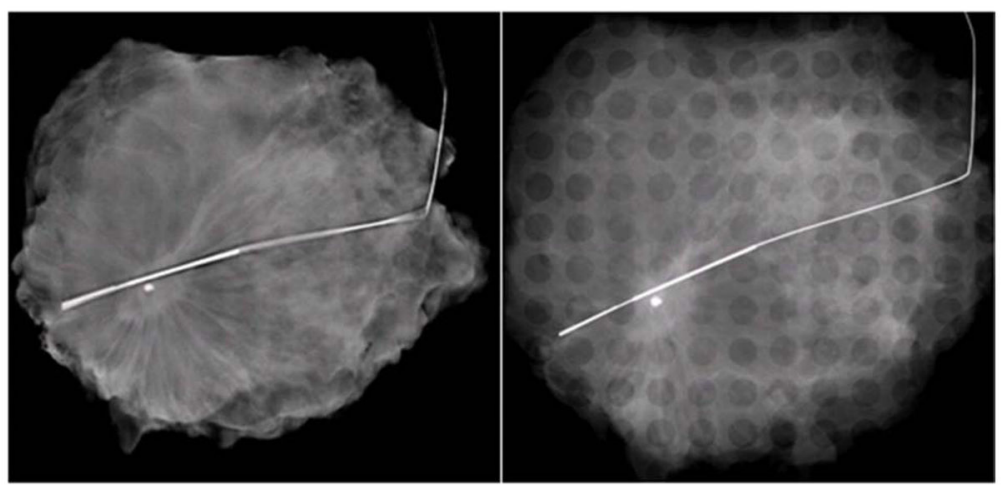

Figure 3.

Left - Reconstructed slice of a specimen using an s-DBT system. The spiculated margins and architectural distortion are more apparent along all edges compared to the $2 \mathrm{D}$ mammography image of the same specimen (Right). 

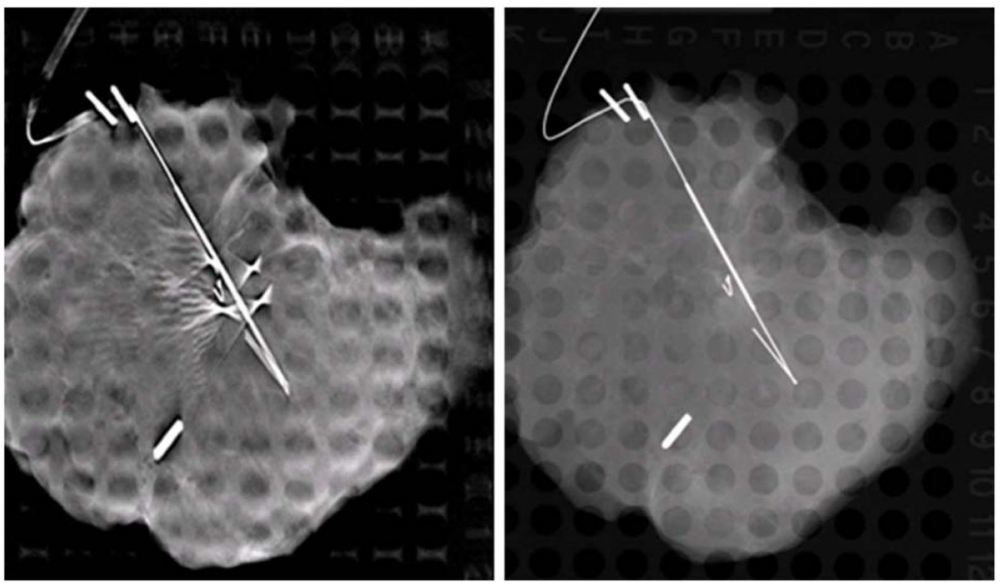

Figure 4.

Left - Reconstructed slice of a specimen using an s-DBT system. Right - 2D mammography image of the same specimen. Biopsy needles are present in the s-DBT reconstructions and not in the 2D mammography image. 


\section{Table 1}

Calculated sensitivity and specificity values by modality and reader. Values were calculated from malignancy scores. Malignancy scores from 3 to 5 were considered positive for disease.

\begin{tabular}{|c|c|c|c|c|}
\hline & \multicolumn{2}{|c|}{ Sensitivity } & \multicolumn{2}{c|}{ Specificity } \\
\hline Reader & FFDM & s-DBT & FFDM & s-DBT \\
\hline $\mathbf{1}$ & $24 / 24(1.00)$ & $23 / 25(0.92)$ & $4 / 14(0.29)$ & $2 / 13(0.15)$ \\
\hline $\mathbf{2}$ & $21 / 25(0.84)$ & $19 / 25(0.76)$ & $5 / 14(0.36)$ & $7 / 14(0.50)$ \\
\hline $\mathbf{3}$ & $24 / 25(0.96)$ & $25 / 25(1.00)$ & $0 / 13(0.00)$ & $0 / 13(0.00)$ \\
\hline $\mathbf{4}$ & $23 / 25(0.92)$ & $25 / 25(1.00)$ & $4 / 14(0.29)$ & $2 / 14(0.14)$ \\
\hline
\end{tabular}

FFDM = full field digital mammography modality

s-DBT = stationary digital breast tomosynthesis modality 


\section{Table 2}

Results of McNemar's test for discordance between the two modalities for all four readers. Insignificant discordance was found between the two modalities for readers 1, 2, and 3. In the case of reader 4, diagnostics based on two modalities are likely to be different.

\begin{tabular}{|c|c|c|c|}
\hline Reader $1(p$-value $=0.5637)$ & Modalit & $=s-D B T$ & \\
\hline Modality=FFDM & Negative & Positive & Sub-total \\
\hline Negative & 1 & 1 & 2 \\
\hline Positive & 2 & 32 & 34 \\
\hline Sub-total & 3 & 33 & 36 \\
\hline
\end{tabular}

\begin{tabular}{|c|c|c|c|}
\hline Reader $2(p-$ value $=0.2482)$ & Modali & $=\mathrm{S}-\mathrm{DBT}$ & \\
\hline Modality=FFDM & Negative & Positive & Sub-total \\
\hline Negative & 5 & 4 & 9 \\
\hline Positive & 8 & 22 & 30 \\
\hline Sub-total & 13 & 26 & 39 \\
\hline
\end{tabular}

\begin{tabular}{|l|c|c|c|}
\hline Reader 3 (p-value=NA) & \multicolumn{2}{|c|}{ Modality=s-DBT } & \\
\hline Modality=FFDM & Negative & Positive & Sub-total \\
\hline Negative & 0 & 0 & 0 \\
\hline Positive & 0 & 35 & 35 \\
\hline Sub-total & 0 & 35 & 35 \\
\hline
\end{tabular}

\begin{tabular}{|c|c|c|c|}
\hline Reader $4(p$-value $=0.0455)$ & Modality & $=\mathrm{s}-\mathrm{DBT}$ & \\
\hline Modality=FFDM & Negative & Positive & Sub-total \\
\hline Negative & 2 & 4 & 6 \\
\hline Positive & 0 & 33 & 33 \\
\hline Sub-total & 2 & 37 & 39 \\
\hline
\end{tabular}

FFDM $=$ full field digital mammography modality $\mathrm{s}$

DBT $=$ stationary digital breast tomosynthesis modality 


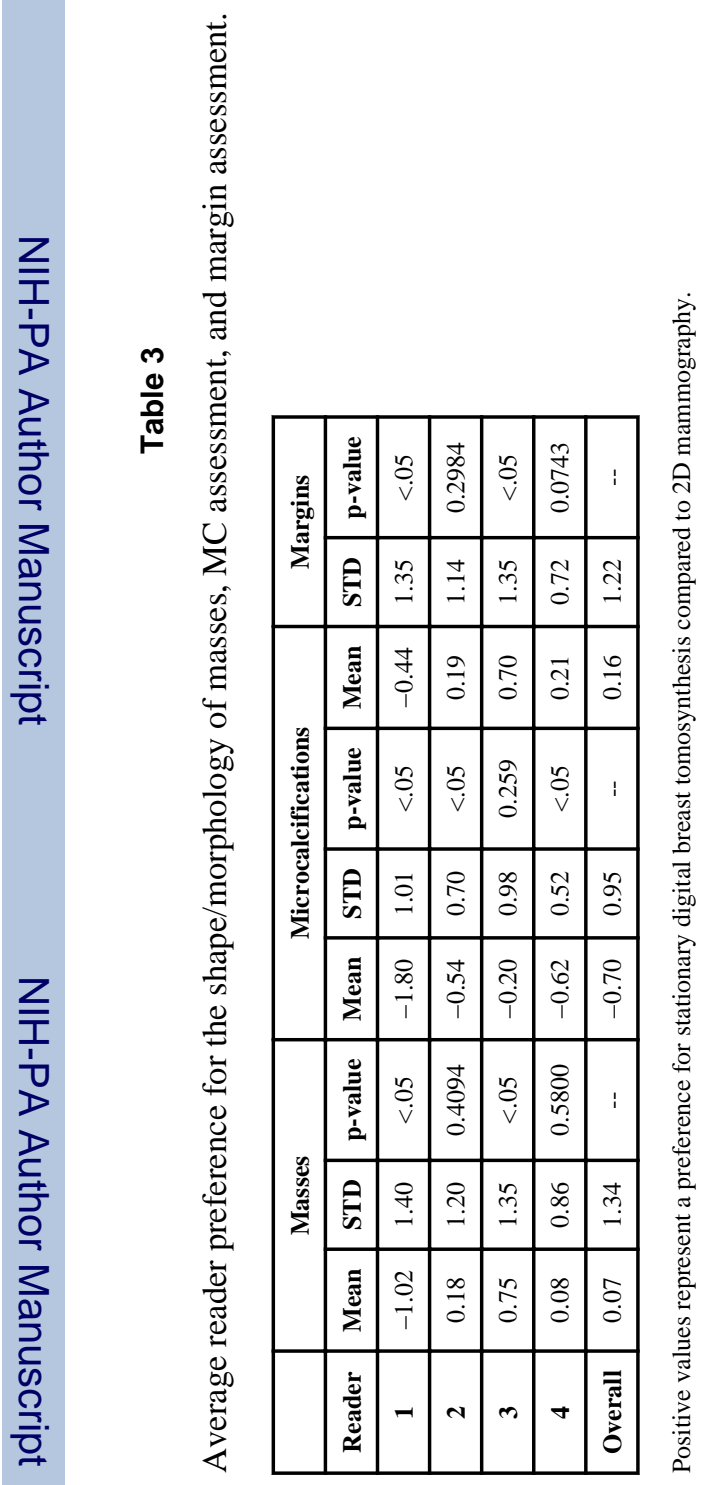

Acad Radiol. Author manuscript; available in PMC 2015 December 01. 


\section{Table 4}

Results of the secondary analysis performed on the preference portion of the reader study. It was tested whether the mean preference was larger than zero using a linear mixed model with a random intercept effect and Wald test.

\begin{tabular}{|c|c|c|c|}
\hline & Grand Mean Estimate & Standard Error & Two-sided p-value \\
\hline Shape/morphology & 0.0598 & 0.1416 & 0.6751 \\
\hline Microcalcifications & -0.6718 & 0.1030 & $<0.05$ \\
\hline Margins & 0.1586 & 0.1422 & 0.2718 \\
\hline
\end{tabular}

Positive values represent a preference for stationary digital breast tomosynthesis compared to $2 \mathrm{D}$ mammography. 